\title{
Advent of The New Media: The influence of intrinsic and extrinsic motivation in online photo sharing behaviours on Facebook among young Malaysian adults
}

\author{
Wan Ching Lee ${ }^{1}$, Thinavan Periyayya ${ }^{2 *}$, and Nair $\mathrm{GV}^{3}$ \\ ${ }^{1}$ Universiti Tunku Abdul Rahman, Faculty of Creative industries
}

\begin{abstract}
Social networking sites have become an important channel of communication in people's daily lives. Online photo sharing, a type of artefact sharing on Facebook has also grown in popularity. Thus, the purpose of this research is to study the relationship of intrinsic and extrinsic motivations in online photo sharing behaviours on Facebook. The study will also examine privacy concerns regarding online photo sharing. A non-random purposive sampling approach gathered a total of 422 Malaysian young adults aged between 18 and 34. Data was collected via a web based questionnaire distributed on Facebook. The results show that intrinsic and extrinsic motivations have positive correlations with online photo sharing behaviours on Facebook. Intrinsic motivations showed a stronger connection to online photo sharing behaviours. There was no significance found between privacy concerns and online photo sharing behaviours on Facebook. There was also no difference between genders in their online photo sharing behaviours. However, males showed higher privacy concerns in regards to online photo sharing on Facebook.
\end{abstract}

\section{Introduction}

Online social networking sites have gained tremendous popularity around the world over the past few years [1-2]. Facebook is the most popular social network, and has surpassed the 1 billion mark of users worldwide as of March 2015 [3-4]. Results from a recent study showed that Facebook is also the most popular social networking site in Malaysia [2]. Online photo sharing is one of the top applications used on Facebook [1], and in Malaysia, 88\% of Malaysian online consumers upload their photos on social networks such as Facebook [5]. Majority of Facebook users worldwide, as well as Malaysia, are young adults in the age group of 18 to 34 years old [6]. Generally, reports show women are more active on social media as well as in online photo sharing [7].

In several studies on online sharing behaviours, theories such as the motivation theory of self-determination, social exchange theory, social capital and the social cognitive theories were drawn on to help explain individuals' motivations to share. Researchers have found that intrinsic and extrinsic motivations both act as a crucial determinant in knowledge and artefact sharing behaviour on online social networks [8]. This study also seeks to investigate how privacy concerns of users affect their online photo sharing behaviour.

\section{Problem statements}

A key question would be what motivates Malaysian netizens to be so heavily invested in such photo sharing behaviours. Sharing photos online is one of the most popular activities on Facebook [5], and is also rapidly growing among Malaysian Facebook users [2-6]. Research has also been limited to certain aspect of motivations and has so far only focused on the Flickr online community in the United States [9-10]. Thus far, there is no published research on what motivates users to share their photos on Facebook. The lack of data regarding online photo sharing behaviors on Facebook in the Malaysian context provides an opportunity to explore and contribute new information to this area of study, as geographical and cultural differences may result in different findings [11]. The impact of privacy concern and photo-sharing behaviour on Facebook has not been researched yet and as such this aspect will also be explored in this study.

\footnotetext{
* Corresponding author: thinavan@utar.edu.my
} 


\section{Research questions}

Based on the problem statement the following research questions were formulated to guide this study:

- What is the relationship between intrinsic motivations and online photo sharing behaviours on

- Facebook among young Malaysian adults?

- What is the relationship between extrinsic motivations and online photo sharing behaviours on Facebook among young Malaysian adults?

- Which motivational factors (Intrinsic or extrinsic motivations) are stronger in users' online photo sharing behaviour on Facebook among young Malaysian adults?

- What is the relationship between online privacy concerns and online photo sharing behaviours on Facebook among young Malaysian adults?

- Is there a gender difference among young Malaysian adults' online photo sharing behaviours on Facebook?

- Is there a gender difference among young Malaysian adults' online privacy concern and online photo sharing behaviours on Facebook?

\section{Literature review}

In online social networks, the content is completely dependent on its creation by the users themselves [10], thus, without user content sharing, it would be meaningless to be a member of such sites. Sharing can have separated into two distinct forms namely, knowledge sharing, and artefact sharing [10]. Knowledge sharing is written information to be shared on one's network. This has been widely studied. Artefact sharing focuses on the sharing of personal content such as photos and videos where content created may or may not be shared. Studies on Artefact sharing is lacking and thus, this study aims to close this gap and contribute data that would continue to create a more comprehensive understanding of the motivating factors that drive users to share online photos on Facebook.

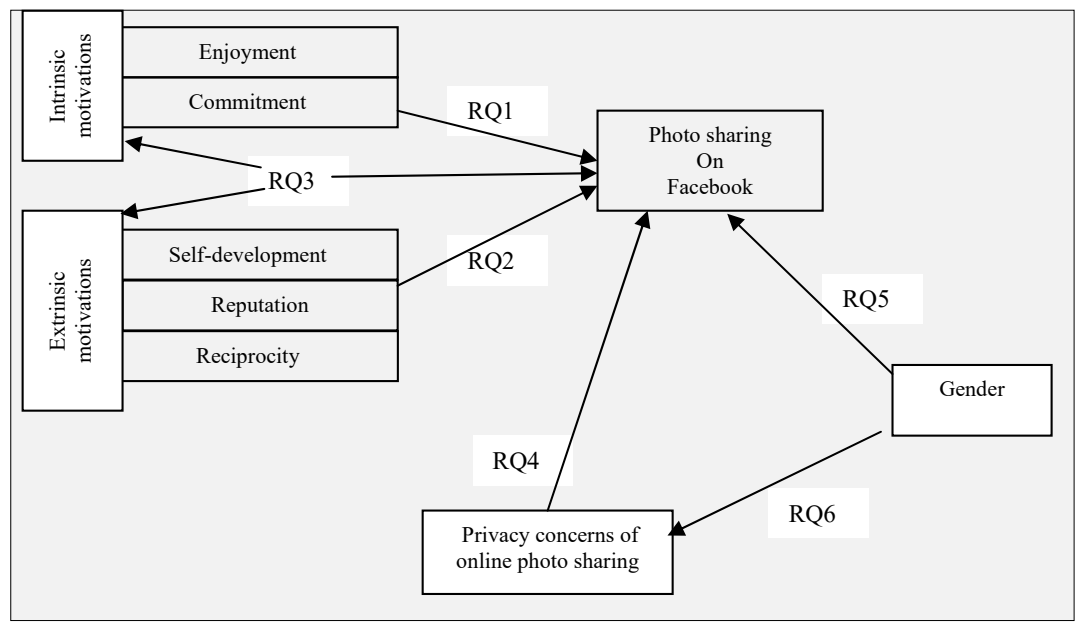

Fig.1. Conceptual Framework

The conceptual framework in Fig 1 is constructed based on various theoretical perspectives such as the self-determining theory of motivation [12], the social exchange theory [13], as well as the social capital theory [14]. These theoretical perspectives were applied to many studies that focused on the motivations behind online sharing behaviours of internet communities. Thus, this study also draws the strengths of these theoretical perspectives and focuses them on the research questions of this study.

Previous studies have shown that intrinsic motivations such as enjoyment and commitment play a crucial role in motivating users to share online. Enjoyment was found to have a positive correlation to share knowledge and content in online communities such as open source soft wares and online communities such as Wikipedia [15-16]. Artefact sharing communities such as Flickr was also noted to be influenced by the motivation of enjoyment $[9,10,17]$. The intrinsic motivation of commitment was positively correlated to both quality and quantity of photo sharing on an online community on Flickr [9-10]. Based on this it can be hypothesized that there is a significant relationship between intrinsic motivation and young Malaysian adults' online photo sharing behaviour on Facebook.

Several studies also looked into the relationship between extrinsic motivations (self-development, reputation, and reciprocity) and photo sharing behaviour. It was also found that social network users were more likely to continue using the social networks when other users reciprocated positive encouragement. Several studies similarly showed that 
software contributors placed greater emphases on self-development and reputation-gaining $[15,18,19]$. In one study it showed that users rated the motivation of reputation as a strong motivator for their friends to share information on Facebook [17]. Therefore, it can be hypothesized that there is a significant relationship between extrinsic motivation and young Malaysian adults' online photo sharing behaviour on Facebook.

Research question three aims to find out if intrinsic or extrinsic motivations play a stronger role in user's online photo sharing behaviour. One study showed that intrinsic motivations were more significant while in another [15] it leaned towards extrinsic motivations [19]. Given the mixed results it can be hypothesized that there is a significant difference in the influencing role of intrinsic and extrinsic motivations in young Malaysian adults' online photo sharing behaviour on Facebook.

Research question four aims to investigate the relationship between users' privacy concern and how it influences their online photo sharing behaviours on Facebook. Prior studies concluded that there is an inconsistency between users' privacy concern and their behaviour. Participants with profiles on social networking websites were less concerned with identity information disclosure and allowed anyone to view their profile without toggling their privacy setting [20]. Based on this Hypothesis four was formulated: that there is a significant relationship between privacy concern and young Malaysian adults' online photo sharing behaviour on Facebook.

Gender differences in online photo sharing behaviours are also evident in previous researches. In general, studies have shown that females posted more photos than males [7,21,22].On privacy concern, male participants showed greater risk taking attitudes and were less concerned with identity information disclosure where else women showed more concern over their privacy, had less friends but still were more likely to share [20].Based on this hypothesis five and six was formulated that is, there is a significant gender difference in young Malaysian adults' online photo sharing behaviour on Facebook; and that there is a significant difference between males and females on privacy concern and young Malaysian adults' online photo sharing behaviour on Facebook.

\section{Method}

The online questionnaire for this quantitative study targeted members of Facebook in Malaysia. Non-probability purposive, convenient sampling was used to obtain the participants for this study. The multi-step distribution procedure, also known as the snowball sampling method was used to collect the required data. The online survey with regards to the 2013 version of Facebook was conducted until enough participants were reached, and the duration of this exercise was from November 2013 to April 2014. The online questionnaire consisted of a total of 59 questions. All the scales used in this study were validated in the original studies, and were validated again in the present study. The variables of the study consist of the independent variables of intrinsic motivation (enjoyment and commitment), extrinsic motivation (self-development, reputation, and reciprocity), and the dependent variable is Facebook users' online photo sharing behaviours. Users' online privacy concern will be an independent variable to see if it impacts the dependent variable that is photo sharing behaviours on Facebook. SPSS was used to run statistical analysis and tests such as Pearson R, multiple regressions, and independent $\mathrm{T}$ test was used to test the hypotheses. The pilot test conducted showed that the reliability of the scales was all above 0.70 for the Cronbach's alpha. Out of 501 respondents, a total of 422 valid participants were used in this study.

\section{Results}

\subsection{Demographic profile \& Facebook usage}

Out of 501 respondents, a total of 422 valid participants were used in this study. This was a response rate of $84 \%$. The participants' age was between 18 and 34 years old $(\mathrm{M}=24, \mathrm{SD}=3)$. Most of the participants' current residence was in Malaysia (94.1\%). Female participants accounted for 59.2\% while male participants accounted for $40.8 \%$. A total of $77 \%$ of the participants were university students; $30.3 \%$ young working adults; $2.8 \%$ of the participants self-employed and $6.2 \%$ unemployed.

Majority of the participants, $43.6 \%$, have been Facebook members between four to five years and another $40.3 \%$ members of Facebook for six to seven years. A total of $7.6 \%$ of them have been members of Facebook for eight to nine years and only $6.4 \%$ were members for less than three years. In terms of friends the users' had on Facebook, $94.2 \%$ of the participants had more between 500-900 friends in their profile. On the question of usage of Facebook on a typical weekday, $73.2 \%$ of participants spent between one to five hours on Facebook while $14.5 \%$ of the participants spent between five to ten hours on Facebook. A total of $3.8 \%$ spent more than 10 hours on Facebook and only 1.2 $\%$ of them spent less than an hour on Facebook.

Number of photos shared. Participants were asked on how many photos they have currently shared on Facebook. A total of $29.1 \%$ of the participants shared 100 to 499 photos on Facebook; $18.2 \%$ of them shared photos in the range of 500 to 999 photos; $17.3 \%$ had shared less than a 100 photos on Facebook; $15.9 \%$ of the participants shared between 1000 and 1499 photos on Facebook;7.3\% shared about 1500 to 1999 photos;2.8\% responded that they have shared between 2000 and 2499 photos, and another $2.8 \%$ shared between 3000 and 3499 photos; $2.4 \%$ shared between 2500 and 2999 photos and $4.0 \%$ of them shared more than 3500 photos. Respondents were also asked on the frequency of the type of photos (Events, self-portrait, friends, family, pets/animals, scenery, and objects) they would usually share on 
Facebook. For events, $37.7 \%$ of the respondents said they would often share photos of events on Facebook, 35.1\% responded sometimes, $13.5 \%$ responded always, $11.4 \%$ responded rarely, and $2.4 \%$ responded never. Self-portrait photos were less frequently shared as $41.7 \%$ of them said they would rarely share photos featuring themselves only, $29.6 \%$ responded sometimes, $14 \%$ responded often, $10.7 \%$ responded never, and a small percentage of $4 \%$ responded always. The frequency of sharing photos on family, pets and animals, scenario and objects were not popular.

\subsection{Results on Hypothesis Testing}

Results for hypotheses one showed a significant positive correlation between intrinsic motivations and online photo sharing behaviour $(r=.60, p<.001)$. Both the intrinsic motivation of enjoyment $(r=.58, p<.001)$ and commitment $(r=$ $.47, p<.001)$ showed a significant positive correlation with online photo sharing behaviours on Facebook. For Hypothesis two, there was significant positive correlation between online photo sharing behaviour and extrinsic motivations $(r=.40, p<.001)$. The extrinsic motivations namely self-development $(r=.34, p<.001)$, reputation $(r=.41$, $p<.001)$, and reciprocity $(r=.32, p<.001)$, showed significant positive correlation with online photo sharing behaviours on Facebook. Results for hypothesis three showed that the intrinsic motivation of enjoyment, $\beta=.44, t(421)=9.82, p<$ .001 , and commitment $(B=.19, t(421)=3.64, p<.001)$ were stronger predictors compared to extrinsic motivations of self-development, $\beta=.013, t(421)=.25, p=.806$, reputation, $\beta=.12, t(421)=1.98, p=.048$, and reciprocity, $\beta=-.023, t$ $(421)=-.43, p=.669$. Hypothesis four showed that there was no significant correlation between photo sharing behaviour and privacy concerns $(r=.06, p=.181)$. The results for hypothesis five showed that there was no significant gender difference between males $(M=16.13, S D=3.34)$ and females $(M=16.10, S D=3.33)$ in their online photo sharing behaviour on Facebook. Results for hypothesis six showed that there was a significant difference between males and females in their online photo sharing behaviours and privacy concerns on Facebook, $\mathrm{t}(420)=-2.31, p=0.21$. Males $(M=60.98, S D=12.78)$. The results are summarised below.

Table 1. Summary of results from Hypothesis testing

\begin{tabular}{|l|l|}
\multicolumn{1}{|c|}{ Hypothesis } & Results \\
\hline $\begin{array}{l}\text { H1: There is a significant relationship between intrinsic motivation and young Malaysian } \\
\text { adults' online photo sharing behaviour on Facebook. }\end{array}$ & Supported \\
\hline $\begin{array}{l}\text { H2: There is a significant relationship between extrinsic motivation and young Malaysian } \\
\text { adults' online photo sharing behaviour on Facebook. }\end{array}$ & Supported \\
\hline $\begin{array}{l}\text { H3: There is a significant difference in the relationship strength between intrinsic and } \\
\text { extrinsic motivation in young Malaysian adults' online photo sharing on Facebook. }\end{array}$ & Supported \\
\hline $\begin{array}{l}\text { H4: There is a significant relationship between privacy concern and young Malaysian adults' } \\
\text { online photo sharing behaviour on Facebook. }\end{array}$ & Not Supported \\
\hline $\begin{array}{l}\text { H5: There is a significant gender difference in young Malaysian adults' online photo sharing } \\
\text { behaviour on Facebook. }\end{array}$ & Not Supported \\
\hline $\begin{array}{l}\text { H6: There is a significant difference between males and females for privacy concern and } \\
\text { young Malaysian adults' online photo sharing behaviour on Facebook. }\end{array}$ & Supported \\
\hline
\end{tabular}

\section{Discussion}

Results supported hypothesis one and the findings are consistent with previous study done on knowledge sharing behaviours as well as online photo sharing behaviours where enjoyment and commitment were found to be positive correlated to knowledge sharing in online communities [15-16]. This positive correlation with intrinsic motivations and online photo sharing behaviour suggests that young Malaysian adults are highly motivated intrinsically to share their photos and enjoy doing this; they also feel committed and obligated to share photos to keep their online community updated.

Hypothesis two on extrinsic motivation and online photo sharing behaviour was consistent with the findings from previous research. Findings in this research were similar to previous research that investigated artefact sharing in online communities such as Flickr and YouTube. Research findings also linked that the trade-off between quality and quantity in online photo sharing in which users who were motivated by self-development and reputation were more concerned about the quality of their photos [9]. Earlier research results showed that sharing YouTube videos were strongly correlated with attention received by others which could be translated to the intrinsic factors namely self-development, reciprocity and reputation [23].

For hypothesis three, intrinsic motivations were stronger predictors due to the nature of the online social network of Facebook. Different social networks cater to different types of users, as well as different types of users could be present in the online network itself and hence results may vary from different social networks [10]. The type of online 
social network that is social in nature and have known social connections on a more personal level, as is the case of Facebook, users in this network indulge in sharing photos for intrinsic rewards.

Hypothesis four was rejected as there was no significant relationship between the online privacy concerns and online photo sharing behaviours on Facebook. Higher privacy concerns did not lead to an increase of online photo sharing behaviours on Facebook, and neither did it deter them from sharing photos. The results are in line with prior research that has noted the inconsistency between users' privacy concern and their behaviour [17,24,25]. The lack of privacy concern of users was reasoned that the perceived benefits of using the online social networking site and how deeply Facebook has integrated into users' daily routine as an indispensable tool to maintain one's social capital and to maintain communication through technology outweighs the risks of sharing personal content, and users often regarded risk and negative consequences as a third person phenomenon [25].

Hypothesis five was rejected as there was no significant gender difference in regards to their online photo sharing behaviour on Facebook. Prior research noted that females posted more photos than males $[7,21,22,26]$. However, in another study it was reasoned that the similarity across genders in online behaviours may be because that social networking sites may be a place where the creation of mini-cultures, in which separate set of social norms in an online environment are formed with the effect of neutralizing any gender differences [8].

Hypothesis six is accepted as there is a significant difference between male and female in regards to their online privacy concerns on online photo sharing behaviour. In which, research results showed that males are more concerned in regards to their online privacy on Facebook compared to females. Prior research reported that generally there is an inconsistency between users' privacy concerns and their actions [17,20,24,25].

\section{Limitations and Recommendations}

This study used convenient sampling and therefore the results cannot be generalized. It is premature to generalize the findings of this study to other population groups unless the results can be replicated. For future research, the use of qualitative approach should be considered to further enhance the findings of this research. A qualitative research would be able to delve deeper into individual perspectives and motivations to indulge in photo sharing.

\section{Implications of study}

Researches and practitioners can use the data to better understand users' behaviours and how it affects users' personal life, their community, as well as provide further insights on how to improve human interaction, communication and the social bond that society strives on. Designers of such online social networking sites could use this data to explore ways to make the users photo sharing experience more enjoyable and feel more strongly attached to the community to encourage more online sharing behaviours.

1. ComScore,Facebook passes Yahoo! Now the third most trafficked website worldwideRetrieved from http://www.ditii.com(2011)

2. S.E.Mustafa, A.Hamzah,Int. J. of Soc. Science and Humanity, 1, 2 (2011)

3. Statista,Global social networks ranked by number of users. Retrieved from http://www.statista.com(2015)

4. Statista,Active social media penetration in Asian countries. Retrieved from http://www.statista.com 2015)

5. TNS, Largest ever digital research project reveals major changes in online behaviour around the world. Retrieved from http://www.tnsglobal.com(2010)

6. Socialbakers, Heart of Facebook statistics: Facebook statistics by country. Retrieved from http://www.socialbakers.com(2013)

7. A.N Joinson,'Looking at,' 'looking up' or 'keeping up with' people? Motives and uses of Facebook. Proceeding of twenty-sixth annual SIGCHI conference on Human factors in computing system, Italy (2008)

8. N.J.Hum, P.E. Chamberlin, B.L. Hambright, A.C. Portwoord, A.C. Schat, J.L. Bevan, Comp. in Human Behav.27, 5, (2011)

9. O. Nov, C Ye, Why do people share photos online? Antecedents of photos' quality and quantity. Proceedings of the Americas Conference on Information Systems (AMCIS 2009), San Francisco, (2009)

10. O.Nov, M, Naaman,C. Ye, C. Proceedings of AAAI International Conference on Weblogs and Social Media (ICWSM, 2009), San Jose, CA. (2009)

11. Y.Kim, D. Sohn, D, S.M.Choi,Comp. in Human Behav. 27, 1 (2011)

12. E.L. Deci, R.M. Ryan,Intrinsic motivation and self-determination in human behavior. (Plenum, New York, 1985)

13. P.M. Blau, Exchange and Power in Social Life. (Wiley, New York, 1964)

14. J. Nahapiet, S. Ghoshal, S. J Acad. Mgmt.23, 2 (1998)

15. K.Lakhani, R.Wolf, Why hackers do what they do. In J.Feller, B.Fitzgerald, S. Hissam, K. Lakhani, (Eds.), Perspectives in free and open source software. 3-22. (MIT Press, Cambridge, MA, 2005)

16. O. Nov, Comm. ACM, 50, 11 (2007)

17. A. Acquisti, R. Gross, Proceedings of 6th Workshop on Privacy Enhancing Technologies,36-58, (Robinson College, Cambridge, UK, 2006)

18. S. Oreg, O. Nov,Comp. in Human Behav., 24, 5 (2008) 
19. M Wasko, M. S. Faraj, MIS Quarterly, 29, 1 (2005)

20. J.Fogel, E. Nehmad,Comp. in Human Behav. 25, 1 (2009)

21. A.M. Kimbrough, R.E. Guadagno, N.L. Muscanell, J.Dill, Comp. in Human Behav. 29, 3 (2013)

22. A.Mendelson, Z.Papacharissi,Look at us: Collective narcissism in college student Facebook photo galleries. In Z. Papacharissi, (Ed.), The networked self: Identity, community and culture on social network sites, 151-173, (Routledge, New York, 2010)

23. B.A. Huberman, D.M. Romero, F. Wu,J. Info. Science, 35, 6 (2008)

24. S.Ahern, D. Eckles, N. Good, S,King, M. Naaman, M., R. Nair, Proceedings of the SIGCHI conference on Human factors in computing systems, 7 (ACM, USA, Press 2007)

25. B.Debatin, J.P.Lovejoy, A. Horn, B.N. Hughes, J. Comp. Mediated. Comm.15, 1 (2009)

26. A. Lenhart, M. Madden, Teens, privacy, \& online social networks. (Pew Internet and American Life Project Report, 2007) 